\title{
Multi-Objective Optimization of Periodical Objectives within a Process
}

\author{
Moses Oyaro Okello \\ Self-Employed Gulu, Uganda \\ e-mail: mosesokellomoses@gmail.com
}

\begin{abstract}
Multi-objective optimization (MOO) is an optimization involving minimization or maximization of several objective functions more than the conventional one objective optimization, which is useful in many fields. Many of the current methodologies addresses challenges and solutions that attempt to solve simultaneously several Objectives with multiple constraints subjoined to each. Often MOO are generally subjected to linear inequality, equality and or bounded constraint that prevent all objectives from being optimized at once. This paper reviews some recent articles in area of $\mathrm{MOO}$ and presents deep analysis of Random and Uniform Entry-Exit time of objectives. It further break down process into sub-process and then provide some new concepts for solving problems in MOO, which comes due to periodical objectives that do not stay for the entire duration of process lifetime, unlike permanent objectives which are optimized once for the entire process duration. A methodology based on partial optimization that optimizes each objective iteratively and weight convergence method that optimizes sub-group of objectives are given. Furthermore, another method is introduced which involve objective classification, ranking, estimation and prediction where objectives are classified based on their properties, and ranked using a given criteria and in addition estimated for an optimal weight point (pareto optimal point) if it certifies a coveted optimal weight point. Then finally predicted to find how far it deviates from the estimated optimal weight point. A Sample Mathematical Tri-Objectives and Real world Optimization was analyzed using partial method, ranking and classification method, the result showed that an objective can be added or removed without affecting previous or existing optimal solutions. Therefore suitable for handling time governed MOO. Although this paper presents concepts work only, it's practical application are beyond the scope of this paper, however base on analysis and examples presented, the concept is worthy of igniting further research and application.
\end{abstract}

Keywords: Optimization, Multi-Objective Optimization, Decision-Making, Time

\section{Introduction}

To introduce Multi-Objective Optimization, individual terms are first define. So what is Optimization? Optimization is define as finding or making the best effective use of situation or resources available. An Objective is simply define as a goal or aims. Then, Constraint is simply limitation or restriction.

Therefore, Overall Multi-Objective Optimization can be define as an approach for seeking best resources to attain desire goals whether in the present of constraint(s) or not.

Optimization of process is of paramount eminence in many fields such as Science, Engineering, Technology and many more. Its application ranges from biological process Optimal Control, Chemical industrial control to physical process control, Data Science and many more. Optimization succor in abridging cost and wastage of resources and minimizes time for process execution within given constraints to meet desire objectives as illustrated in many articles presented in [1][2][3] and [4]. Take for example a case in a flight or marine vessel, which is required to maximize the amount of load (goods) it carries meanwhile minimizing it weight and size. Some common approach is to minimize the amount of fuel it uses, as reduction in fuel use is directly proportional to decrease in overall weight of a vessel and ultimately the size of the fuel tank. Nevertheless, how does one reduce fuel without affecting the distance to be cover by the vessel? Some engineers would use the shortest path/rout possible for navigation; others might use the approach of redesigning an energy efficient engine to ensure minimum consumption and minimum wastage of fuel [5] during navigation. All these are targeting at minimizing cost and wastage of resources such as fuel.

Another example, in chemical industrial setting, where the objective is to maximize the product of reaction. Some engineers would device a methodology by maximizing rate of reaction but some reaction are endothermic which requires too much input energy to run the reaction process fully and efficiently. So how does one minimize energy inputs required to run such endothermic reaction meanwhile maximizing product of the reaction? All these are some of the notable situation that requires good optimization strategy. 
A simple System often consists of a single objective and perhaps a single constraint and they are linear normally. However a system start getting complicated when it's non-linear and in addition, when there are more than one or many constraints and many or multiple objectives to be solve simultaneously. Such systems are quite not straightforward to optimize due to their Complexity.

In MOO, Objectives can have influence over other Objective, this make it difficult to solve such problem. I.e. the influence can be either negative or positive.

A negative influence is when solving Objective results in degrading the optimal solution of at least one or more of the other remaining Objectives in MOO. Therefore, this mean to obtain the best optimal solution of such objective, the remaining objective may have poor optimal solution or even worst solution or vice versa.

This is often referrers to as non-dominated, non-inferior, Pareto efficient, or Pareto optimal if not any of the objective values can be upgrade without devaluing some of the other objective values.

Most challenges in multi-objective optimization often referred to as multi-criteria programming are generally subjected to linear inequality, equality and or bounded constraint(s) that prevent all objectives from being simultaneously solve for instance a case where number of objectives are more than that of controllable variable or, perturbation that generates uncertainties. Many scholars have put several approaches and model for finding optimal weight point of multi-objective optimization in place. The methods addresses several challenges in multi-objective optimization and their application in many field such as science, finance, engineering and many more. For instance in field of electricity in electrical power balance where trade-off between voltage and electrical grid requirement [6] and where demand of electricity and electricity generation needs to be balance with considering constraints bonded to it. The challenges involve in multi-objective optimization is finding an optimal weight point solution thought there may exist multiple solution for a given multi-objective optimization problem, however the problem is finding such solution which is not simple such as that of a single objective optimization.

The following functions are use throughout the entire text to give the general problem definition see (1) extracted from article by [17]

$$
\begin{array}{cc}
\min f_{k}(X) & k=1, \ldots, K \\
\text { s.t } g_{j}(X) \leq 0, & j=1, \ldots, J \\
h_{m}(X)=0, & m=1, \ldots ., M \\
x_{i}^{L} \leq x_{i} \leq x_{i}^{U}, & i=1, \ldots ., N
\end{array}
$$

Where the following are define in $\mathrm{MOO}$ with total variable $N$, and total Objectives as $K$. In addition, total constraints is defined a as $J$ for inequality, $M$ for equality. In addition, lower $L$ and upper boundaries $U$ for each variable $x_{i}^{L}, x_{i}^{U}$ are define too.

Optimization can be express as minimization of an objective function or maximization of Objective function. However, in most cases such as in (1), it is often express as minimization as many objective are model in term of cost or time. Therefore, minimizing cost and or time mean an optimal performance, which is less costly, or time wasting. For some model of Objective function, may require maximization of Objective function such as maximizing products, maximizing profit etc.

\section{Literature Review}

Here are some of the notable multi-objective optimization methods and their application in many fields such as Science, Engineering, Economics, Data Science and many more. In addition, to currently and often use standard approach by setting a fix optimal weight point among multiple objectives. This paper is a polished and extended version of an article which initially appeared in preprint [7], which presented some of review in Multi-Objective Optimization field. MOO approaches are categories into four major categories [8] and [9] as below i.e. No preferences, Priori method (lexicographical programming, Goal programming, Utility programming), Posteriori Method and Interactive Method.

In the literature review here, in addition to theoretical manuscripts, discussion about some selected application of Multi-Objective Optimization such as in Data Science, Engineering, and Economics etc.

A paper in [10], introduces an approach based on transformation of multi-stage optimal control model (OCM) with random switching time. They separated the problem into two sub-domain i.e. optimal control with random time horizon and Multi-stage Optimal Control Model (OCM).

In their model and reformulation as deterministic (OCM), they assume that switching time divides the time horizon that is define as stage 1 and stage 2 or even more by random variable $\tau$ out of sample space $\Omega=[0, \infty]$. They further 
applied reformulation problem introduced in by [11] about deterministic Optimal Control Model with infinite time horizon and they got the results (2) and (3) and there are probability at some point that the switch has not occurred as they pointed out.

With

$$
\begin{gathered}
\max _{u(t)} \int_{t_{0}}^{\infty} e^{-\rho t} z 1(t)\left[g 1(x(t), u(t), t)+\eta(x(t), u(t), t) V^{*}(x(t), t)\right] d t \\
s . t \quad \dot{x}=f_{1}(x(t), u(t), t), \quad x\left(t_{0}\right)=t_{0} \\
z 1(t)=-\eta(\dot{x}(t), u(t), t), \quad z 1\left(t_{0}\right)
\end{gathered}
$$

$$
\begin{aligned}
V^{*}((t), t) & =\max _{u(t)} \int_{t}^{\infty} e^{-\rho(s-t)} g 2(x(s), u(s), s, x(t), t) d s \\
\text { s.t } \dot{x}(s) & =f_{2}(x(s), u(s), s, x(t), t) \quad x(t) \lim _{t^{\prime}>t} \varphi\left(\left(t^{\prime}\right)\right)
\end{aligned}
$$

A case study by [12] showed their method base on genetic gender approach for solving multi-objective optimization challenges of detection observers. In their method, the previous knowledge about a single gender of all included solutions is use for making difference among groups of objectives. The knowledge is from fitness of a single person and used during current parental crossover in evolutionary multi-objective optimization process.

An approach by [13] on multi-objective optimization model for sizing and operation optimization district heating system with heat storage tanks. The model includes process design and energy integration method for optimizing the temperature interval, the volume and the operation strategy of thermal storage tanks.

The application of multi-objective optimization in water distribution system by [14], they use Ant-colonyOptimization for the optimization algorithm which concern with water quality base objectives in Water Distribution System design alongside other common objectives.

A multi-objectives decision support system developed for rehabilitation planning of public infrastructure by [15]. Their method provides decision makers a collection of optimal rehabilitation tradeoff over a preferred analysis period. They handled two main objectives function cost and performance at once, together with the collection of attached constraints. The mechanism is based on a fitness-oriented method where challenges information is taken into account. To further analyze, cost and performance all together, a normalization methods of all objectives is attained through time-value concept for both cost and condition states. Their proposed methodology is based on life-cycle costing approach using a dynamic markov chain to constitute the degeneration methodology and optimal rehabilitation profile is found using algorithm.

A study by [15] on Multi-Objective Bayesian Optimization (MOBO) problem presented a framework for taking user choices in (MOBO). They called the improvement approach as Expected Weighted Hypervolume Improvement (EWHI). They defined the hyper-volume of the dominated region based on continuous unlike lesbegue. Sampling approximation method was also used for EWHI computation as the EWHI take a form of integral in which no close form expression exist in general case.

They demonstrated this on a Bi-Objective Optimization problem (4) where there is preference over one Objective and in addition, there is preference over a certain region.

$$
\left\{\begin{array}{l}
f_{1}\left(x_{1}, x_{2}\right)=\frac{1}{15} e^{-\frac{x_{1}}{15}, \frac{1_{[0,150]}\left(x_{1}\right)}{150} \cdot \frac{1_{[0,60]}\left(x_{2}\right)}{60}} \\
f_{2}\left(x_{1}, x_{2}\right)=\frac{1}{2}\left(\varphi\left(x, \mu_{1},\right)+\varphi\left(x, \mu_{2}, C\right)\right)
\end{array}\right.
$$

Where $\varphi\left(x, \mu_{1},\right)$ denotes Gaussian probability density function, for means and covariant matrix are denoted as $\mu$ and $C$ respectively evaluated at $x$. According to them, $f_{1}(x)$ is entirely based on user preference.

[17] presented some work in Multi-Objective Optimization in Python, which solve challenges that most framework in Python programming Language does not address such issue. They developed pymoo, a multi-Objective Optimization framework in Python. They implemented their framework that can be modified by user by inputting custom operators. Furthermore, sample test Challenges are presented too where gradients can be retrieved by automatic differentiation out of the box.

Here are some of the common methods often use in solving Multi-Objective Optimization problems, $\varepsilon-$ Constraint Method: In the constraint method, one of the Objective is optimized but the remaining of the objectives are set as the constraints within the user preference see (5) extension from (1)

$$
\min f_{q}(X) \leq \varepsilon_{\mu}, \quad k=1, \ldots, K, \quad k \neq q
$$




$$
\begin{array}{cc}
\text { s.t } g_{j}(X) \leq 0, & j=1, \ldots, J \\
h_{m}(X)=0, & m=1, \ldots, M \\
x_{i}^{L} \leq x_{i} \leq x_{i}^{U}, & i=1, \ldots \ldots, N
\end{array}
$$

The method can be used in either convex or non-convex MOO problems. However, there is need to take care while choosing $\varepsilon-$ vector to ensure that it is within the range of minimum and maximum values of the individual objective function.

Weighted Sum Methods: in this method, all the objectives in the MOO, or set of Objectives are first scalarize into a single objective by summing each objective pre-multiplied by user defines or supplied weight. See (6)

$$
\begin{array}{cc}
\text { minmize } F(x)=\sum_{k=1}^{K} w_{k} f_{k}(x) \\
\text { s.t } g_{j}(X) \leq 0, \quad j=1, \ldots, J \\
h_{m}(X)=0, \quad m=1, \ldots, M \\
x_{i}^{L} \leq x_{i} \leq x_{i}^{U}, \quad i=1, \ldots \ldots, N
\end{array}
$$

Weighted Metric Method: This is another method just like weighted sum method, this one add up all the multiple Objective based on weighted distance metric of any solution from the ideal solution $z^{*}$ see (7) for the expression of such condition.

$$
\begin{gathered}
l_{p}(x)=\left(\sum_{k=1}^{K} w_{k}\left|f_{k}(x)-z_{k}^{*}\right|^{p}\right)^{\frac{1}{p}} \\
\text { s.t } g_{j}(X) \leq 0, \quad j=1, \ldots ., J \\
h_{m}(X)=0, \quad m=1, \ldots, M \\
x_{i}^{L} \leq x_{i} \leq x_{i}^{U}, \quad i=1, \ldots \ldots, N
\end{gathered}
$$

Several applications of multi-objective optimization problem has been extensively use in many fields for example in large scale clustering presented in articles in [16][18] and in some of the field like the one presented on the paper [19]. In conclusion, many scholars propose several methods; however, some method might be feasible for one or more situations while in another situation it might not be perfectly feasible.

\section{Multi-Objective Optimization Proposed Concepts}

This portion discusses, analyzes and presents some of the challenges and solution involve in multi-objective optimization, which do not appear in single objectives optimization, and then it gives ways forward in solving those problems.

First is to classify objectives base on how long they take in a process lifetime during process execution. It is classified into two categories as describe here below.

\section{Permanent/Long-Term Objectives}

Permanent or long-term objective is define as the one that stay or is needed from the starts of a process execution up to the end of process execution that does not result in redesigning the optimal weight point since the optimal weight point remains the same. For instance, if a given process starts at time $\left(t_{0}\right)$ and ends at time $\left(t_{n}\right)$, permanent objectives also starts at time $\left(t_{0}\right)$ and ends at time $\left(t_{n}\right)$ as the process.

\section{Periodical/Short-Term Objectives}

Unlike in Permanent or Long-term objective in multi-objective optimization, a temporal objective is the one that is needed only for a particular period or for a short while, less than the time for process execution and not throughout the entire process. Periodical objectives can pop in or out at any moment during process execution time, these could be due to perturbation or any other factor which may cause it, for example consider the objectives function given below (8) in $\mathrm{MOO}$

$$
\min \left(f_{1}(x), f_{2}(x) \ldots f_{k}(x)\right)
$$

Where integers, $k \geq 2$ are the number of objectives. Out of total objective $f_{k}(x)$, one or more might be very crucial from the start of the process or in the middle or even towards the end of process execution but not throughout the entire process execution and somewhere somehow, the periodical objective will be no longer relevant once it is not needed. 
This periodical objectives which switch on and off, may results in the entire multi-objectives optimization solution being solve again and again, with and without the periodical objective, when in and out of the process execution as the optimal weight point of multi-objectives varies and are not the same when one include or remove a given objective from many objectives.

For example, the optimal weight feasible range $x^{*}$ of $W_{1} \neq W_{2}$ of the equation in multi-objective (9) and (10) are not the same because of the absent of the objective, in other word, $W_{1}$ is not an optimal weight point of equation (10) whose optimal weight point is $W_{2}$ and $w:=\left[f\left(x^{*}\right), x^{*}\right]$

$$
W_{1}:=\min \left(f_{1}(x), f_{2}(x), f_{3}(x), f_{4}(x), f_{5}(x)\right)
$$

The MOO (9) contains five objectives functions that need to be minimize.

$$
W_{2}:=\min \left(f_{1}(x), f_{2}(x), f_{3}(x), f_{4}(x), f_{5}(x), f_{6}(x)\right)
$$

In addition, the MOO (10) contains six objectives functions not the same as equation (9) however all the five objectives are the same with additional objective.

These gives challenge of redesigning another new optimal weight point, which is optimal for the remaining objectives, which are only relevant during execution excluding the objective that is no longer needed, or including the additional objectives as in equation (10).

\section{Random Entry-Exit Time}

Here Objectives are Indexed and ranked base on time in multi-objective optimization when dealing with shortterm/periodical objectives and long-term objectives/permanent objectives mixed altogether within a process lifetime. It is very crucial to index objectives based on time they either enter $\left(t_{i}\right)$ or exit $\left(t_{i}{ }^{\prime}\right)$ a process for all the objectives involve for easy ranking, classification, and solving using partial optimization technique presented here. Given time series for entry time $T=\left\{t_{0}, t_{1}, t_{2}, t_{3}, \ldots, t_{n}\right\}$ and exit time as $T^{\prime}=\left\{t_{0}{ }^{\prime}, t_{1}{ }^{\prime}, t_{2}{ }^{\prime}, \ldots, t_{n}{ }^{\prime}\right\}$.

\section{Random Entry Time}

In this case, all objectives are assume to ends at the same time a process terminates however, their entry time into the process varies randomly. Given example of objectives function from (10) are index based on time objectives enter in a process but first their time of entry is estimated.

Supposed the following estimated time for objectives in (10) are recorded, such that $f_{1}(x)$ enter at $t_{0}, f_{2}(x)$ enter at $t_{6}, f_{3}(x)$ enter at $t_{3}, f_{4}(x)$ enter at the same time as $f_{2}(x)$, and $f_{5}(x)$ enter at $\left(t_{7}\right)$

Indexing the five objectives as below:

$$
\left(f_{1}(x)\right)_{t_{0}},\left(f_{2}(x)\right)_{t_{6}{ }^{\prime}}\left(f_{3}(x)\right)_{t_{3}},\left(f_{4}(x)\right)_{t_{6}{ }^{\prime}}\left(f_{5}(x)\right)_{t_{7}}
$$

Rearranging (11) in ascending order of entry time such that objective that enter first is rank first and given high priority, and those that enter last are given last priority or ranked last. However for two or more objectives with same time of entry are ranks using any other criteria such as dependency on other conditions to give them high priority. If no such or any other condition exist, those objective with same entry time can be put at any order see condition (12) after rearranging.

$$
\left(f_{1}(x)\right)_{t_{0}},\left(f_{3}(x)\right)_{t_{3}},\left(f_{4}(x)\right)_{t_{6}{ }^{\prime}}\left(f_{2}(x)\right)_{t_{6}},\left(f_{5}(x)\right)_{t_{7}}
$$

In (12), $f_{4}(x)$ enter at the same time as $f_{2}(x)$, however it is rank first than $f_{2}(x)$ as it is assumed that $f_{2}(x)$ is dependent on $f_{4}(x)$. Incase such condition do not exist, and then their order of ranking won't be an issue.

\section{Random Exit Time}

Here, all objectives are assumed to enter at the same time when process execution begins however, their exit time out of the process varies. Given example of objectives function from (12) are indexed base on time objectives exit a process but first their time of exit out of the process is estimated.

Supposed the following estimated exit time for objectives in (12) are noted, such that $f_{1}(x)$ exit at $t^{\prime}{ }_{2}, f_{2}(x)$ exit at $t_{6}^{\prime}, f_{3}(x)$ exit at $t^{\prime}{ }_{3}, f_{4}(x)$ exit at the same time as $f_{2}(x)$, and $f_{5}(x)$ exit at $t^{\prime}{ }_{7}$

Indexing the five objectives as below:

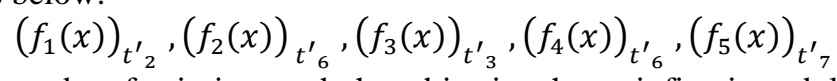

Rearranging (13) in descending order of exit time such that objective that exit first is rank last and given low priority, and those which exit last are given high priority or ranked first. However for two or more objective with same time of exit are ranked using any other criteria such as dependency on other conditions, to give them high priority. If no such or any other condition exist, those objectives with same exit time can be put at any order see condition (14) after rearranging.

$$
\left(f_{7}(x)\right)_{t^{\prime}{ }_{2}},\left(f_{4}(x)\right)_{t^{\prime}{ }_{6}},\left(f_{2}(x)\right)_{t^{\prime}{ }_{6}},\left(f_{3}(x)\right)_{t^{\prime}{ }_{3}},\left(f_{1}(x)\right)_{t^{\prime}{ }_{2}}
$$


In (14), $f_{4}(x)$ exit at the same time as $f_{2}(x)$ however, it is ranked first than $f_{2}(x)$ as it is assumed that $f_{2}(x)$ is dependent of $f_{4}(x)$. Incase such condition do not exist, then their order of ranking won't matter.

\section{Mixed Random Entry-Exit Time}

In this scenario, it is based on the idea that in multi-objective optimization some objectives have random entry and exit time in that an objective can enter at any time and exit at any time before the process terminate and for all objectives in a process, their entry and exit times are scatter randomly within the process. Also considering this process also contains permanent objectives. It is like a combination of objective with varying entry-exit time.

Take for instance the case below:

$$
\left\{\begin{array}{l}
f_{1}(x) \Rightarrow \operatorname{enter}\left(t_{0}\right) \rightarrow \operatorname{exit}\left(t^{\prime}{ }_{2}\right) \\
f_{2}(x) \Rightarrow \operatorname{enter}\left(t_{4}\right) \rightarrow \operatorname{exit}\left(t^{\prime}{ }_{6}\right) \\
f_{3}(x) \Rightarrow \operatorname{enter}\left(t_{3}\right) \rightarrow \operatorname{exit}\left(t^{\prime}{ }_{3}\right) \\
f_{4}(x) \Rightarrow \operatorname{enter}\left(t_{6}\right) \rightarrow \operatorname{exit}\left(t^{\prime}{ }_{6}\right) \\
f_{5}(x) \Rightarrow \operatorname{enter}\left(t_{7}\right) \rightarrow \operatorname{exit}\left(t^{\prime}{ }_{7}\right)
\end{array}\right.
$$

For case above with different entry-exit time, there are two scenario prior to ranking using time of entry or exit.

We consider Exit time $\left(T^{\prime}\right)$ and Entry time $(T)$ so we rank them by taking which is more important, Entry time or Exit time. If we priorities Entry time $(T)$, then we can rank them base on entry time only and ignore exit time $\left(T^{\prime}\right)$, See (11) where it is ranked base on entry time so exit time is of less or no priority.

However, for the case when exit time is of high priority, entry time is ignored. See (14). Many approaches can be use more efficiently for this special case.

\section{Uniform Exit-Entry Time}

In this case, all objectives, exit at $\left(t_{i}^{\prime}\right)$ and another enter at $\left(t_{i}\right)$ in a process at the same time; one or more time in a process $P(Y)$ noting that $t_{i}^{\prime}=t_{i}$. The question is how to handle this scenario. One of the way presented here is to split the process into multi-Process referred to as sub-process $p(y)$ within a process. Given that $\left\{p_{1}(y), p_{2}(y), p_{3}(y), \ldots, p_{n}(y)\right\} \in P(y), y \in Y$ and parameter $(y)$ of the process represents the objectives to be executed within a process $y=\left\{f_{1}(x), f_{2}(x), f_{3}(x) f_{4}(x), f_{5}(x) \ldots . f_{k}(x)\right\}$. Where the objective ends, a new one starts. In addition, each sub-process is solve separately by using the presented methods just normally like a full objectives within a process.

However splitting a process into a sub-process does not means terminating the entire process. The previous subprocess terminates and the new sub-process continuous instantaneously as the previous sub-process exits. For example of two sub-process $p_{1}(y), p_{2}(y)$ see (15) where objectives $f_{1}(x), f_{2}(x), f_{3}(x)$ all terminates at time $t_{6}^{\prime}$ and objectives $f_{4}(x), f_{5}(x)$ begins at time $t_{6}$ but as you know $t_{6}=t_{6}^{\prime}$

$$
P(y)=\left\{p _ { 1 } ( y ) \left\{\begin{array}{l}
f_{1}(x) \Rightarrow \operatorname{enter}\left(t_{0}\right) \rightarrow \operatorname{exit}\left(t^{\prime}{ }_{6}\right) \\
f_{2}(x) \Rightarrow \operatorname{enter}\left(t_{4}\right) \rightarrow \operatorname{exit}\left(t^{\prime}{ }_{6}\right) \\
f_{3}(x) \Rightarrow \operatorname{enter}\left(t_{3}\right) \rightarrow \operatorname{exit}\left(t^{\prime}{ }_{6}\right)
\end{array} \mid p_{2}(y)\left\{\begin{array}{l}
f_{4}(x) \Rightarrow \operatorname{enter}\left(t_{6}\right) \rightarrow \operatorname{exit}\left(t^{\prime}{ }_{8}\right) \\
f_{5}(x) \Rightarrow \operatorname{enter}\left(t_{6}\right) \rightarrow \operatorname{exit}\left(t^{\prime}{ }_{10}\right)
\end{array}\right\}\right.\right.
$$

Given that $t_{i}=t_{i+1}$

Now for the first sub-process can be group, index using random Entry-Exit time method for ranking and solve by either partial optimization or Objective Classification, ranking, Estimation and Predictive measurement presented here below.

The second sub-process also follows the procedure for the first one and it continues for all the sub-process up to the last one.

\section{Partial Optimization Concept in Multi-Objective Optimization}

In response to the challenges due to the presents of periodical objective in multi-objective optimization, I present some concepts that is about partial optimization of many objectives in multi-objective optimization; two initial idea related are presented.

\section{Iterative Multi-Level Approach}

The approach is iterative in that, it involves taking two or more solvable set from multiple objectives set and their optimal weight $W_{q}$ for the sub-set solve. This solution becomes or is set as constraints of the next solvable set or objectives from the multiple set. It is done iteratively until all objectives are finished and the final optimal weight point is assumed the most optimal weight among multi-objectives, which favor the objective with the highest priorities. 
Consider objective function (16) or recall the equation from (1).

$$
\min \left(f_{1}(x), f_{2}(x), f_{3}(x), \ldots, f_{k}(x)\right)
$$

In addition, integers $k \geq 2$ are the number of objectives set $X$ are input variables of objective function. However, element $x^{*} \in X$ is further defined as feasible solution or feasible decision of an objectives vector.

$$
W:=\left[f\left(x^{*}\right), x^{*}\right] \in \mathbb{R}^{k}
$$

Initially, first take the second objective function $f_{2}(x)$ and set the previous (first) objective function $f_{1}(x)$ as a constraint. Find the optimal weight $W_{0}$ as their optimal weight between the two objectives just like the one presented in a paper in [20] which attempt to solve two or more objective by setting one objective as a constraint and another objective to be minimize. However, the difference in their method is that when solving many objective, one of the objective is optimized meanwhile the rest of the remaining objectives are set as constraints. For this partial optimization, an objective is optimized and their optimal weight feasible range is set as a constraints of the next objective to be optimized in the MOO. This is done iteratively until all objectives are finished.

Please note (18) shows the condition is also subjected to constraints either inequality, equality and or bounded constraint as explained in (1).

$$
\begin{gathered}
W_{0}:=\min \left(f_{2}(x)\right) \\
\text { s.t } f_{1}(x) \leq \varepsilon_{q}, g_{j}(X) \leq 0, \quad j=1, \ldots, J \\
h_{m}(X)=0, \quad m=1, \ldots, M \\
x_{i}^{L} \leq x_{i} \leq x_{i}^{U}, \quad i=1, \ldots \ldots, N \\
W_{l} \leq \varepsilon_{1}, \quad l=1, \ldots, L
\end{gathered}
$$

Next iteration is to take objective function $f_{3}(x)$ and set the previous optimal weigh $W_{0}$ for objectives function $f_{1}(x)$, $f_{2}(x)$ as a constraint to find optimal weight $W_{1}$. See (19).

We set $W_{0}$ as constraints of the currently being optimized function simply to ensure that the currently being optimized function should not interfere with already optimized function (weight).

$$
\begin{gathered}
W_{1}:=\min \left(f_{3}(x)\right) \\
\text { s.t } W_{0} \leq \varepsilon_{1}
\end{gathered}
$$

The process continues until all the functions in multi-objectives $f_{k}(x)$ are finished and the final weight $W_{\mu}$ is assumed the optimal weight for all objectives. An overall formulation is as below (20):

$$
\begin{gathered}
W_{q}:=\min \left(f_{q+2}(x)\right) \\
\text { s.t } W_{q-1} \leq \varepsilon_{q}
\end{gathered}
$$

However, alternatively instead of using the method presented in (18) for solving bi-objective optimization, the first objective is optimized as single objective optimization problem as below (21):

$$
\begin{gathered}
W_{0}:=\min \left(f_{1}(x)\right) \\
\text { s.t } g_{j}(X) \leq 0, \\
h_{m}(X)=0,
\end{gathered}
$$

So followed by second optimization while setting first optimal weight as constraint to second optimization see (22)

$$
\begin{gathered}
W_{1}:=\min \left(f_{2}(x)\right) \\
\text { s.t } W_{1} \leq \varepsilon_{0}
\end{gathered}
$$

The process continues until all the functions in multi-objectives $f_{k}(x)$ are finished and the final weight $W_{\mu}$ is assumed the optimal weight for all objectives. An overall formulation is as below (23):

$$
\begin{gathered}
W_{q}:=\min \left(f_{q+1}(x)\right) \\
\text { s.t } W_{q} \leq \varepsilon_{q-1}
\end{gathered}
$$

\section{Weight Convergence Optimization}

The second approach is by dividing a given set of objectives into several small sub-sets of objectives, which is easily solvable without much burden, and each sub-set, solve separately see (24). Let $W_{q, i}$ where $\mu$, and $i$ are integers of weight level and set of weight respectively. Weight Level zero $W_{0, i}$

$$
\min \left(\begin{array}{c}
W_{0,0}\left(f_{1}(x), f_{2}(x), f_{3}(x), \ldots, f_{k 1}(x)\right), W_{0,1}\left(f_{k 1+1}(x), f_{k 1+2}(x), f_{k 1+3}(x), \ldots, f_{k 2}(x)\right), \ldots \\
\ldots, W_{0, i}\left(f_{k n+1}(x), f_{k n+2}(x), f_{k n+3}(x), \ldots \ldots, f_{k}(x)\right)
\end{array}\right)
$$




$$
\begin{gathered}
\text { s.t } W_{0, i} \leq \varepsilon_{0} \\
k 1<k 2<k n<k ; n \in \mathbb{N}
\end{gathered}
$$

The process continues up to a set of weight with a single element (weight point) $W_{q, 0}$ and that is assume to be an optimal weight point for multi-objective optimization see (25). Weight Level $\mu$ for $W_{q, 0}$

\section{Objectives Classification, Ranking, Estimation, Prediction}

$$
\begin{gathered}
\min \left(W_{q, 0}(\ldots \ldots), W_{q, 1}(\ldots \ldots), W_{q, 2}(\ldots \ldots), \ldots . W_{q, i}(\ldots \ldots)\right) \\
\text { s.t } W_{q-1, i} \leq \varepsilon_{q}
\end{gathered}
$$

The second approach, which is based on the followings, objective classification, Ranking, Estimation, and Predictive measurement to find how far a system will deviates from a preferred optimal weight. This is in the present of a decision maker (DM), where there could be preference.

The following steps are how the process can be executed in order to find an optimal weight point.

\section{Step 1: Objectives Classification}

Several criteria can be used to classify objectives. For instance, each objective can be categories as independence and dependence objective. Independence is an objectives which does not rely on other objectives as oppose to dependence objective, which depend on other objectives in other word solving one objective, affect the other which is dependent on it also known as Pareto Optimal solution which means a solutions that cannot be improve without degradation of at least some of the solutions. Another way of classifying objectives is by categorizing them into either temporary or permanent objective as discussed above. This is mainly to priorities and takes care of objectives such that when dynamically choosing weight, such objectives are taken care of.

\section{Step 2; Objective Ranking}

In this step 2 of objective ranking, in a set of multi-objectives, objectives are first rank in order of preference or merit base. Second, each individual participating objective their minimum or maximum optimal weight point is determined such that when finding weight such points are taken care of. Furthermore, objective can be rank in order with which they exit process execution, starting with the one that exit earliest being the last to be optimize and the one that exit last being the first to be optimize in partial optimization. The same applies to the order with which Objectives enter into a process, i.e. those that enter first are rank first up to the one that enter last into the process.

For instance, $x_{i}^{L}$ and $x_{i}^{U}$ are defined as lower and upper bound limits or constraints see(1) for both minimum and or maximum optimal weight point for a given single objective see condition (26) or from condition (1).

Step 3: Objective Estimation

$$
x_{i}^{L} \leq x^{*} \leq x_{i}^{U}
$$

In estimation stage, this requires prior knowledge of objectives ranking and classification, then an optimal weight point is first estimated that certifies the ranking and classification in step 1 and 2.

\section{Step 4: Objective Prediction.}

This step is based on the principle of predictive measurement in that control variable can be predicted base on prior and current state, and hence a control strategy is use such that a controller parameter is adjusted accordingly to meet the estimated weight point of multi-objective optimization base on step 1 and 2 .

Suppose $\delta d$ is deviation between estimated desired optimal weight $W_{e}$ and predicted optimal weight $W_{p}$ and in addition $W_{e}$ and $W_{p}$ if are quantifiable i.e. can be express numerically as express in (27):

$$
\delta d=\left|W_{e}-W_{p}\right|
$$

As $\delta d \rightarrow 0$, the better as the condition can be express as $0 \leq \delta d \leq \Delta$ where $\Delta$ is deviation limits within which $\delta d$ is unacceptable and an appropriate strategy needs to be put in place to drive the system towards zero i.e. $\delta d \leq \Delta$.

The best predicted optimal weight occurs when $\delta d=0$ or when $\left|W_{e}-W_{p}\right|=0$. Although theoretically it is convincing and achievable, in practice it could be very difficult to reach $\delta d=0$, however $\delta d \leq \Delta$ is fine.

\section{Objective Alignment in Partial Optimization.}

In the above method presented under partial optimization, Objectives has to first be align in order to make it easily possible to remove an objective or many objective without needs to redesign the entire solution. This is purposely to give temporary objective less priority and permanent objective very high priority.

\footnotetext{
a. Classification.
} 
First before beginning to optimize the entire multi-objective using method of either iterative or weight convergence, it is first classified into either periodical or permanent objectives. This is to give temporary objective less priority and permanent objective very high priority

\section{b. Ranking}

After classifying the objective, it is further ranked base on the time the objectives takes to exit the process before the process execution ends, then optimization is done in a way that objective which are classified as permanent are optimized first without any preference. In addition, the one that are classified as temporary or periodical objective are then optimized later by ranking them in the order with which they enter or exit the process and the one that exit earliest or enter last will be optimized last such that removing will not affect or need to redesign the entire equation. See equation below (28) and (29) where $f_{5}(x)$ is removed and the previous weight $W_{2}$ automatical become the weight of the current equation without the present of the objective function $f_{5}(x)$.

$$
W_{3}:=\min \left(f_{1}(x), f_{2}(x), f_{3}(x), f_{4}(x), f_{5}(x)\right)
$$

The MOO (28) contains five objectives function, which need to be minimized.

$$
W_{2}:=\min \left(f_{1}(x), f_{2}(x), f_{3}(x), f_{4}(x)\right)
$$

The same applies to weight convergence method where permanent objectives are classified and optimized separately and the one, which are temporary or periodical, are optimize separately.

Then later they are finally optimized such that removing portion of the periodical objectives does not affect the entire solution weight (optimal weight point) and resulting in reo.

\section{Sample Problem}

Here two sample examples are discussed from different point of view such graphical, analytical and the second example is about real world example which involve time governed Multi-Objective Optimization problem with bounded constraints.

\section{Mathematical Optimization Sample Problem}

Example 1:

Consider an example of time governed Tri-Objectives Optimization problems (30) where there are three objectives with bounded unconstraint,

$$
\begin{gathered}
f_{1}(x)=0.5 x^{2}+\sin \left(x^{2}\right) \quad ; \quad t_{0} \rightarrow t_{6}^{\prime} \\
f_{2}(x)=x^{2}-1.4 x-1.5 \quad ; \quad t_{3} \rightarrow t_{6}^{\prime} \\
f_{3}(x)=x^{2}-1.1 e^{-x^{2}}-1 ; \quad t_{0} \rightarrow t_{4}^{\prime} \\
\text { Search domain }-3 \leq x \leq 3
\end{gathered}
$$

From the above, to optimize the three objectives, we first optimize single objective under bounded constraint given above. So since objective $f_{1}(x)$ is the only objective that starts from beginning up to the end of the process. it is given highest priority followed by objective $f_{3}(x)$ which starts from beginning but stop somewhere in the middle of the process, and last is objective $f_{2}(x)$ which enter into the process not from the beginning but in the middle and stops at the end of the process.

First Step:

$$
\begin{aligned}
& w_{0}=\min f_{1}(x) \\
& \text { s.t }-3 \leq x \leq 3
\end{aligned}
$$

Therefore, $w_{0}:=\left[f_{1}\left(x^{*}\right), x^{*}\right]$ so, $\left[f_{1}\left(x^{*}\right), x^{*}\right]=\left[2.9 e^{-31}, 4.44 e^{-16}\right]$

Which is approximately in the range of $0<x<0.1$ for the feasible solution of the first objective.

This feasible solution range is therefore use to set bounded constraints for the next objective to solve to ensure that the feasibility range of the first optimized objective is protected.

Second Step:

$$
\begin{gathered}
w_{1}=\min f_{3}(x) \\
\text { s.t } \quad 0<x<0.1
\end{gathered}
$$

So, since $f_{3}(x)$ is next to be optimized, $w_{1}:=\left[f_{3}\left(x^{*}\right), x^{*}\right]$ so, $\left[f_{3}\left(x^{*}\right), x^{*}\right]=\left[-2.1,3.998 e^{-5}\right]$ Which is approximately in the range of $0<x<0.1$ for the feasible solution of the first objective.

These feasible solution range is therefore use to set bounded constraints for the next objective to be solve to ensure that the feasible range of the second optimized objective is protected. However if we are to keep the bound rang of 
the first Objective, $-3 \leq x \leq 3$ one finds that $\left[f_{3}\left(x^{*}\right), x^{*}\right]=\left[-2.1,-3.3307 e^{-16}\right]$ which is still within the range .We take the range of $0<x<0.1$

Third Step:

$$
\begin{gathered}
w_{2}=\min f_{2}(x) \\
\text { s.t } \quad 0<x<0.1
\end{gathered}
$$

Finally, $f_{2}(x)$ the last to be optimize, $w_{2}:=\left[f_{2}\left(x^{*}\right), x^{*}\right]$ so, $\left[f_{2}\left(x^{*}\right), x^{*}\right]=[-1.63,0.1]$.

However if we are to keep the bound range for the first one $-3 \leq x \leq 3$, one finds that $\left[f_{2}\left(x^{*}\right), x^{*}\right]=[-1.99,0.7]$ which makes the feasible solution range of $0<x<0.7$ when the first and the second objectives are not priorities or given higher rank. If given higher rank, so the range has to be in $0<x<0.1$. To ensure that optimal weight point are given respect, favors objectives with higher priority, and not deviated when finding the least objective in the rank. Therefore, any solution in the range of $0<x \leq 0.7$ would be an optimal solution for the three objective.

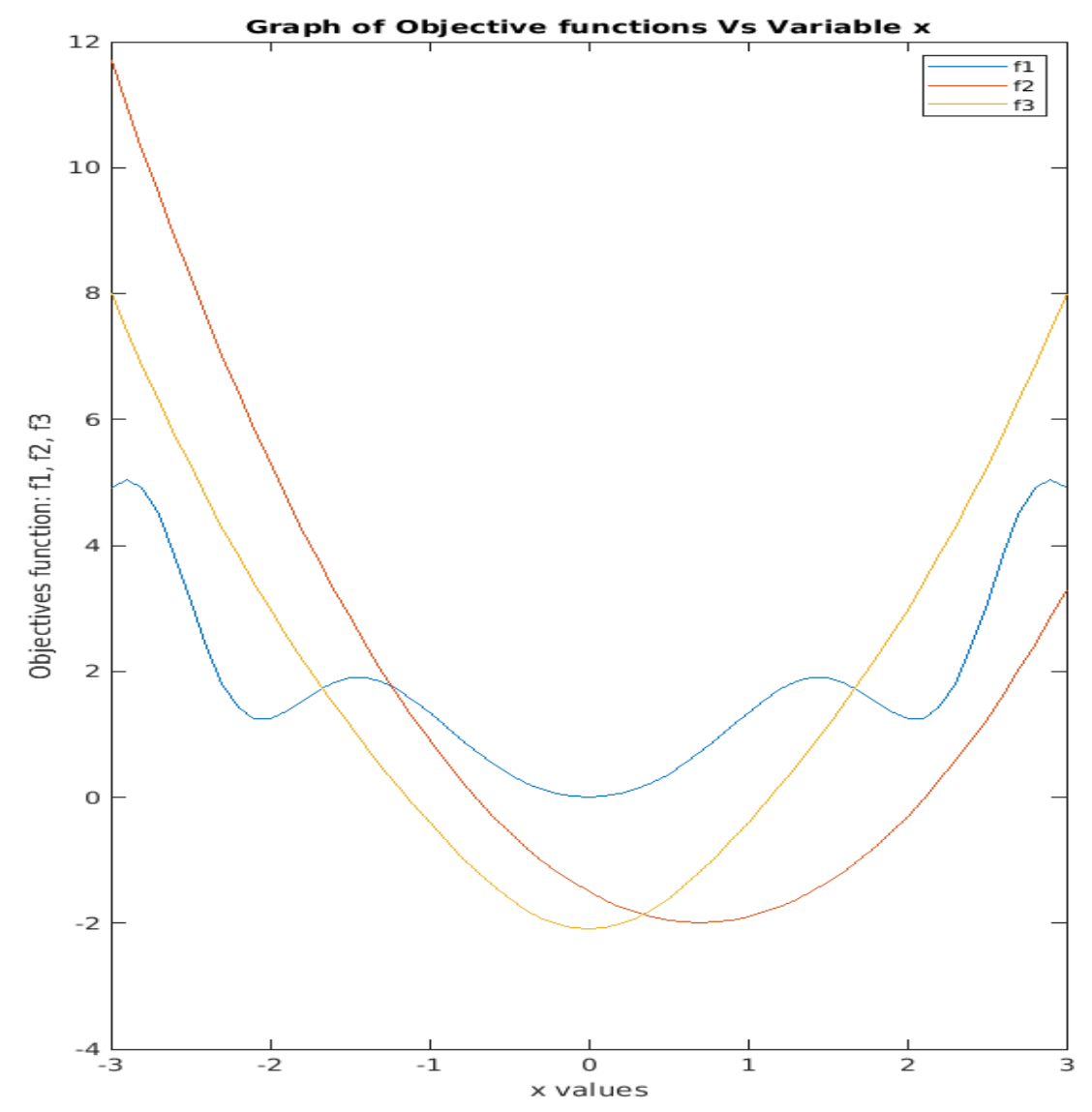

Figure 1: Shows graph of Objectives function (f1, f2, f3) against x variable

Please note an Objective in MOO may be short live (periodical) but still have the highest priority, likewise, an objective may too be long live (permanent) but still have lowest priority.

See below figure 1 shows graph of the three objectives plotted on a graph.

\section{Example 2:}

Another example here in (31) shows MOO with two input variables i.e. $x_{1}, x_{2}$ It is extended from example (30), so reader can try to solve.

$$
f_{1}\left(x_{1}, x_{2}\right)=0.5\left(x_{1}^{2}+x_{2}^{2}\right)+\sin \left(x_{1}^{2}+x_{2}^{2}\right) \quad ; \quad t_{0} \rightarrow t_{4}^{\prime}
$$




$$
\begin{gathered}
f_{2}\left(x_{1}, x_{2}\right)=\left(x_{1}^{2}+x_{2}^{2}\right)-1.4\left(x_{1}+x_{2}\right)-1.5 \quad ; t_{3} \rightarrow t_{6}^{\prime} \\
f_{3}\left(x_{1}, x_{2}\right)=\left(x_{1}^{2}+x_{2}^{2}\right)-1.1 e^{-\left(x_{1}^{2}+x_{2}^{2}\right)}-1 \\
\text { Search domain }-3 \leq x_{1}, x_{2} \leq 3
\end{gathered}
$$

Solutions:

This sample problem is left for reader.

Real World Optimization Sample Problem

Here is another example 3, a Bi-Objective Optimization Problem where first Objective is to minimize time for cargo loading and Offloading on Cargo Ship and the second Objective is to minimize Space in the Cargo Ship by proper arrangement. This is based on Sipping industry where Cargo (Container) ship need to be optimize for transportation. This example is an extracts from published article by (Ajay Menon, Marine Insight, 2021) webpage, and modified in order to fit in this scenario.

Please note: This example 3 may not be a perfect example to depict the situation in Time Governed MOO, but it is an illustration of such scenario, author's attempt to show.

Example 3:

A Cargo (Container) Ship is set to sail from Port A to port C via Port B. The Ship is supposed to load a total of ' $n$ ' Containers from Port A of which half of it i.e $0.5 n$ will be unloaded at Port B and from Port B, another 3 container will be loaded onto the ship for final destination to port $\mathrm{C}(0.5 n+3)$.

1-Design loading arrangement plan of the Containers on the Cargo Ship such that time for transporting is minimized in a way that unloading and loading container at port mid-way to final destination does not result into unloading the entire containers to access those at the bottom, hence causing delays of time and wastage of energy.

2-Minimize space of the Cargo Ship too with the arrangement plan.

\section{a. Unconstraint}

In this part, no condition or constraint is attached.

Solution:

The solution for this is quite straight forward as there is no constraint attached to the container arrangement plan.

Answer: Containers to be transported from Port A to final destination Port $\mathrm{C}$ should be loaded first at the bottom on the Container Ship.

The remaining Containers to be unloaded at mid-way Port B should be loaded on top such that at Port B, unloading them won't affect those at the bottom and won't result into unloading the entire container to access those at the bottom hence minimizing time for unloading and loading.

Please note different arrangement plan can be design for these.

\section{b. Constraint}

The constraint in this case is that the heaviest Containers should be loaded first at the bottom followed by heavier one, up to the lightest containers loaded on top. This is to avoid damaging lighter container when loaded at the bottom and distorting Cargo Ship stability.

If out of the $0.5 n$ containers to be unloaded at port B, about 0.75 of those are containers carrying heavy materials and all the containers to be unloaded at final destination are light containers. In addition, the three Containers to be loaded from port B are very heavy Containers.

Design loading plan for such a Scenario to minimized time for transportation, and empty spaces on the Cargo ship. Solution

Solution is left for reader.

\section{Analysis of the Concepts}

The following analysis attempt to shows the drawback and some advantages of this propose methodology compared to the currently existing methods 


\section{Disadvantage of Existing methods Compared to Presented Concepts}

In most of the current methodologies presented by many scholars tries to solve all multi-objective problem simultaneously which when periodical/short-term objectives is added or remove may result in destabilizing the optimal weight point hence need to redesign the entire solution again. This is not that much problem, however during runtime, it could be tiresome and the hardship to resolve the same thing repeatedly. for example in equation given in (9) and (10) where objective is removed and the entire weight are not the same as (28) and (29).

\section{Advantage of Presented Concepts}

The advantage of this solution presented here is that unlike other existing solutions, partial objective optimization an objective can be remove safely without need to redesign the entire equation as the previous solution become the current solution without need to solve again.

In addition, addition of an objective does affect much, all is needed is to optimize that objective together with existing solution as explained above. However, objective classification and ranking should be carefully done to ensure alignment before optimization.

\section{Conclusion}

Due to ultimatum in seeking the best optimal solution in multi-objective optimization, this paper discusses some notable published paper by scholars, which presents the novel technique in tackling problems in multi-criteria optimization such as evolutionary algorithm methods, flower pollination algorithm and many more.

However, the author further notices some dare in the area of multi-objective optimization where there are situation when one or more objectives are needed either in the beginning or towards the ends or even in the middle of the process execution. The author called this as periodical or temporary objectives or Short term Objective unlike usual objectives that are needed from the start up to the end of process execution. This is called permanent or long-term objectives.

The problem with this is that every time we add or remove an objective from a running process with multiple objectives may results in destabilization of existing optimal weight point as shown in example (9) and (10).

In respond to the challenges, the author presented a theoretical concept, which uses partial optimization iteratively including some methods which use objective classification, ranking, estimation and predictive measurement. These concepts are very convincing theoretically, however their feasibility is far beyond the scope of this paper. Further indepth studies will still be conducted to check the practical application and its feasibility in real world scenario including a numerical simulation or any analytical solution when conducted.

Future research direction: Although this paper presents concepts about partial optimization, and Objective Classification, Ranking, Estimation and Prediction, Further examination or testing of concepts about Estimation and Prediction need to done, as the paper only cover concepts on partial optimization, Classification and, Ranking.

Therefore, Estimation and Prediction remains concepts proposed but their feasibility are still beyond the scope of this paper.

\section{Conflict of Interest}

Author declare that there is no conflict of interest for this manuscript 


\section{References}

[1] Vinter R. Optimal control. Springer Science \& Business Media; 2010 Jun 25.

[2] Lewis, Frank L., Draguna Vrabie, and Vassilis L. Syrmos. Optimal control. John Wiley \& Sons, 2012.

[3] Zhou, Kemin, John Comstock Doyle, and Keith Glover. Robust and optimal control. Vol. 40. New Jersey: Prentice hall, 1996.

[4] Kwakernaak, Huibert, and Raphael Sivan. Linear optimal control systems. Vol. 1. New York:.Wiley-interscience, 1972. Kumar, Devender \& Sharma, Anshul \& Sharma, Somesh. (2012). Developing Model for Fuel Consumption Optimization in Aviation Industry. Innovative Systems Design \& Engineering. 3. 26-36.

[5] Xiao, C.H.A.I., Xiongqing, Y.U. and Yu, W.A.N.G., 2018. Multipoint optimization on fuel efficiency in conceptual design of wide-body aircraft. Chinese Journal of Aeronautics, 31(1), pp.99-106.

[6] Wang Y, Lin X, Pedram M, Park S, Chang N. Optimal control of a grid-connected hybrid electrical energy.Storage system for homes. In2013 Design, Automation \& Test in Europe Conference \&.Exhibition (DATE) 2013 Mar 18 (pp. 881-886). IEEE.

[7] Okello, M. Multi-Objective Optimization Concept Based on Periodical and Permanent Objective within a Process Preprints 2020, 2020050331 (doi: 10.20944/preprints202005.0331.v1).

[8] Sayin S. Kaisa M. Miettinen Nonlinear Multiobjective Optimization Kluwer Academic Publishers.1999 ISBN 07923-8278-1, 320 pages EUR 134.00/USD 129.95. European Journal of Operational Research. 2003;148(1):22930.

[9] Miettinen K, Ruiz F, Wierzbicki AP. Introduction to multiobjective optimization:.Interactive approaches. InMultiobjective Optimization 2008 (pp. 27-57). Springer, Berlin, Heidelberg.

[10] Wrzaczek, Stefan, Michael Kuhn, and Ivan Frankovic. "Using Age Structure for a Multi-stage Optimal Control Model with Random Switching Time." Journal of Optimization Theory and Applications 184.3 (2020): 10651082.

[11] Boukas, El-Kébir, Alain Haurie, and Philippe Michel. "An optimal control problem with a random stopping time." Journal of optimization theory and applications 64.3 (1990): 471-480.

[12] Białaszewski T, Kowalczuk Z. Solving highly-dimensional multi-objective optimization problems by means of genetic gender. InAdvanced and Intelligent Computations in Diagnosis and Control 2016 (pp. 317-329). Springer, Cham.

[13]Fazlollahi S, Becker G, Ashouri A, Maréchal F. Multi-objective, multi-period optimization of district energy systems: IV-A case study. Energy. 2015 May 1;84:365-81.

[14] Shokoohi M, Tabesh M, Nazif S, Dini M. Water quality based multi-objective optimal design of water distribution systems. Water Resources Management. 2017 Jan 1;31(1):93-108.

[15] Farran M, Zayed T. Fitness-oriented multi-objective optimisation for infrastructures rehabilitations. Structure and Infrastructure Engineering. 2015 Jun 3;11(6):761-75.

[16] Feliot, P., Bect, J., \& Vazquez, E. (2018, September). User preferences in Bayesian multi-objective optimization: the expected weighted hypervolume improvement criterion. In International Conference on Machine Learning, Optimization, and Data Science (pp. 533-544). Springer, Cham.

[17]Blank, J., \& Deb, K. (2020). pymoo: Multi-objective optimization in python. IEEE Access, 8, 8949789509.Zhang, Xingyi, et al. "A decision variable clustering-based evolutionary algorithm for large-scale manyobjective optimization." IEEE Transactions on Evolutionary Computation 22.1 (2016): 97-112.

[18] Wang, Rui, Shiming Lai, Guohua Wu, Lining Xing, Ling Wang, and Hisao Ishibuchi. "Multi-clustering via evolutionary multi-objective optimization." Information Sciences 450 (2018): 128-140.

[19] Taboada, Heidi A., Fatema Baheranwala, David W. Coit, and Naruemon Wattanapongsakorn. "Practical solutions for multi-objective optimization: An application to system reliability design problems." Reliability Engineering \& System Safety 92, no. 3 (2007): 314-322.

[20] Yang XS. Flower pollination algorithm for global optimization. InInternational conference on unconventional computing and natural computation 2012 Sep 3 (pp. 240-249). Springer, Berlin, Heidelberg. 